\title{
El falo entre los sexos
}

\section{The phallus between sexes}

\section{Verónica Morelli}

\section{RESUMEN}

Desarrollaremos, en el presente escrito, la función del falo entre los sexos desde el comienzo de la enseñanza de Jacques Lacan hasta el año 1964. Por tal razón, interrogaremos la función del falo significante, precisamente cuando Lacan (1958) enseña que el falo da realidad al sujeto en ese significante e irrealiza las relaciones que han de significarse. A su vez, es también dicho significante el que divide y organiza los roles sexuales.

Argumentaremos la designación de los roles sexuales por el falo significante con la fórmula no ser sin. Lacan (1958/1959) introduce esta fórmula mediante el uso de la negación para situar, en ella, la intervención de la castración.

A causa de la marca que el falo recibe de la castración, este significante tiene una función de equivalencia en la relación con el objeto, la que explicaremos mediante el valor del velo en el amor y sus efectos de comedia. Al placer cómico lo abordaremos, también, en la enseñanza de Freud (1905).

\section{Palabras clave}

Falo - Significante - Castración - Sexo Comedia

\begin{abstract}
In this text, we will develop the role of the phallus between the sexes from the beginning of Jacques Lacan's teaching until 1964. For this reason, we will begin by questioning the role of the phallus signifier precisely when Lacan (1958) explains that the phallus has the role of giving reality to the subject through the signifier and unrealizes the relationships which will be signified. In turn, the signifier divides and organizes the sexual roles.

We will discuss the designation of the sexual roles through the phallus signifier with the formula "being without having". Lacan (1958/1959) introduces this formula by means of denial where he locates castration intervention.

Due to the mark the phallus gets through castration, this signifier has an equivalent role in relation to the object. The previous will be developed by means of the veil value in love and its comedy effects. Comic pleasure will also be approached within Freud teaching (1905).
\end{abstract}

\section{KEYWORDS}

Phallus - Signifier - Castration -

Sex-Comedy 



\section{¿SER Y TENER?}

Iniciaremos el desarrollo por la relación del ser del sujeto con el falo, para introducir su función significante en la asunción de los roles sexuales.

Lacan (1959) formula que el falo tiene relación con el ser del sujeto por su función significante, y ello designa dos posibilidades: ser o tener el falo. En este sentido, la dialéctica del ser y del tener vale tanto para devenir hombre como mujer.

Ahora bien, la función del significante falo introduce una ambigüedad, al constituir al sujeto, que es efecto de la ley y del lenguaje. El falotiene relación con el ser del sujeto porque se trata del significante, dado que los seres hablantes son designados como falo imaginario por el lenguaje, al estar sujeto al deseo del Otro, y a la vez el sujeto no es el falo, porque el discurso lo oculta al operar la interdicción estructural del incesto.

Lacan (1958/1959) en El Seminario. Libro 6. El deseo y su interpretaciónescribe lo siguiente:

El sujeto es y no es el falo. Lo es porque ése es el significante bajo el cual el lenguaje lo designa, y no lo es porque en otro plano el lenguaje, y justamente la ley del lenguaje, se lo sustrae (p.239).

En el momento en que la ley le sustrae al sujeto, en su advenimiento, esa realidad fálica imaginaria se inscribe que él es y no es el falo. Esta operación del lenguaje ya marca cierta elección en torno al ser y tener que sucede en el intervalo entre la "identificación significante y el reparto de los papeles” (Lacan, 1958/1959, p. 239).
Lacan (1958) señala estas funciones del significante en su Escrito "La significación del falo" cuando dice: "Pero se puede, ateniéndose a la función del falo, señalar las estructuras a las que estarán sometidas las relaciones entre los sexos” (p.661).

Y continúa:

Digamos que esas relaciones girarán alrededor de un ser y un tener que, por referirse a un significante, el falo, tienen el efecto contrariado de dar por una parte realidad al sujeto en ese significante, y por otra parte irrealizar las relaciones que han de significarse (Lacan, 1958, p.661).

¿Qué quiere decir Lacan al formular que el falo significante irrealiza las relaciones que han de significarse cuando da realidad al sujeto en el significante?

El falo significante irrealiza las relaciones que han de significarse entre los sexos porque, como dijimos anteriormente, la interdicción del incesto sustrae esa posición fálica imaginaria de ser el significante del deseo del Otro, es decir, lo que da realidad al sujeto. Esta sustracción que la ley produce es la prueba de la "función de nudo" (Lacan, 1958, p. 653) de la castración, porque, entre otras funciones, instala en el sujeto una posición inconsciente, lo divide, a partir de la cual, puede "identificarse con el tipo ideal de su sexo" (p.653). Lo enunciado dice, entonces, que no hay representación de los sexos en el inconsciente.

Que la diferencia sexuada no está representada en el inconsciente, se reitera, cuando, más tarde, Lacan (1964) afirma que "en el psiquismo no hay nada que permita al sujeto situarse como ser macho o 
ser hembra" (p. 212) desde el nacimiento, como sí lo es la designación en el plano biológico. Se trata de una un dilema estructural que deviene del encuentro del sujeto con la privación en el campo del Otro. Privación que instituye el significante, el que introduce que un objeto falta en lo real, y designa la falta de un significante que permita nombrar la oposición masculino-femenina.

Por esta falta de representación en el campo del Otro para nombrar la diferencia sexuada, los seres humanos solo pueden figurar en el psiquismo los ideales viriles y femeninos, y para figurarlos, Lacan (1964) utiliza la noción de "mascarada" (p.201).

La mascarada es una noción que Lacan toma de Joan Riviére, analista inglesa, aunque primero introduce el término "parecer" (Lacan, 1958, p.661), el que, además, revela diferentes comportamientos en la asunción de los roles sexuales.

A propósito de la función del parecer, en su escrito "La significación del falo", Lacan (1958) formula lo siguiente: "Es por la intervención de un parecer que se sustituye al tener, para protegerlo por un lado, para enmascarar la falta en el otro (...) (p. 661). Entonces, parecer, ser o tener, es la manera de significar, mediante la lógica atributiva fálica, la posición sexuada que asume cada sujeto ante la falta en ser. Porque del falo solo hay atributos, ya que dicho significante "no es lo que se tiene ni lo que se es" (Rabinovich, D, 2009, p. 79).

Para argumentar la diferencia sexuada con relación al significante falo y los diferentes comportamientos en la asunción de roles sexuales, alrededor del ser y tener, nos remitimos a la fórmula "no ser sin" (Lacan, 1958/1959, p. 240).
Lacan (1958/1959) por medio de la forma en que opera la negación en el lenguaje, aprehende, en el deslizamiento concerniente al uso del verbo ser en francés, la fórmula no ser sin, y en ella advierte la intervención de la castración. Por eso en El Seminario. Libro 6. El deseo y su interpretación leemos: "En la inflexión del no ser sin, en torno a la asunción subjetiva que oscila entre serlo y tenerlo, que interviene la realidad de la castración” (...) (p.240).

Con respecto a la relación del sujeto masculino con el falo, el momento decisivo donde interviene la castración es que él es y no es el falo, pero "él no es sin tenerlo” (Lacan, 1958/1959, p. 240).

\section{(...) El valor central que adquiere} el falo surge de que, en cierta experiencia, el pene del sujeto ha sido comparado con el objeto, ha tomado cierta función de equivalente o de patrón en la relación con el objeto. $\mathrm{Y}$ en alguna medida puede decirse que, en relación con cierta renuncia a su relación con el falo, el sujeto toma posesión de esa infinitud, de esa pluralidad, de esa omnitud del mundo de los objetos que caracteriza al mundo del hombre (Lacan, 1958/1959, p. 240).

Por lo que se refiere al sujeto femenino, su relación con el falo y la fase fálica se articulan de un modo diferente respecto del hombre. Por eso "La relación del sujeto femenino con el falo tiene por fórmula: ella es sin tenerlo" (...) (Lacan, 1958/1959, p. 240).

La castración es esa inflexión que representa la negación: no ser sin. Mediante esta fórmula Lacan (1958/1959) continúa argumentando lo que el sujeto irreali- 
za por la operación del significante, y al mismo tiempo por su operación resuelve, vía las identificaciones, con el parecer en la asunción de los roles sexuales. Es decir, asumirán una posición masculina, no sin tenerlo, o femenina, en la que lo es, sin tenerlo. Por la castración, la relación de la mujer con el falo es que ella lo es, no siéndolo.

Como consecuencia del encuentro con la castración en el Otro, el sujeto renuncia parcialmente al falo, y por eso cuenta con la posibilidad de entrar en el intercambio.

\section{EL AMOR, LO CÓMICO Y LA RISA ENTRE LOS SEXOS}

Subrayemos que el falo "tiene una función de equivalente en la relación con el objeto" (Lacan, 1960/1961, p. 266).

Una de las equivalencias fálicas es la que se despliega en el plano del amor. En la situación amorosa, el sujeto dibuja una imagen sobre un velo de la que el otro será un equivalente fálico, pero no el falo.

Lacan (1956/1957) trabaja la equivalencia fálica en el plano del amor mediante la función del velo en El Seminario. Libro 4. La Relación de Objeto cuando expone:

El velo, la cortina delante de algo, permite igualmente la mejor ilustración de la situación fundamental del amor. Puede decirse incluso que al estar presente la cortina, lo que se encuentra más allá como falta tiende a realizarse como imagen. Sobre el velo se dibuja la imagen. Ésta y ninguna otra es la función de una cortina, cualquiera que sea. La cortina cobra su valor, su ser y su consistencia, precisamente porque sobre ella se proyecta y se imagina la ausencia (p. 157).

La falta, que se realiza como imagen, revela esa parte fálica que el sujeto resigna, y señala que el falo funciona más allá de la relación entre los sexos. De ahí que lo que se encuentra más allá como ausencia, el falo, se refleja en la imagen del otro que funciona como velo y vela la falta en el sujeto.

Ocupar el lugar de equivalencia fálica en el amor recuerda aquellos efectos que la comedia produce: lo cómico, lo ridículo, como también, la risa al final de la copulación.

Lacan (1958)encuentra que lo cómico resulta de la intervención del parecer, por eso, expresa que dicha sustitución(...) "tiene el efecto de proyectar enteramente en la comedia las manifestaciones ideales o típicas del comportamiento de cada uno de los sexos, hasta el límite del acto de la copulación” (p. 661).

Para desarrollar lo cómico en la manifestación del ideal entre los sexos, abordaremos, en primer lugar, brevemente, la comedia, y en segundo lugar, la diferencia entre el falo significante y los ideales.

Lacan (1957/1958) sitúa en la comedia la función del falo significante, y la distingue de la tragedia en la que el falo se encuentra en su dimensión imaginaria.

En la historia antigua, la comedia (Lacan, 1957/1958, pp. 269-272) vino a representar las relaciones del hombre con la mujer y completa la trilogía clásica de la tragedia antigua. En el plano de la comunidad, la tragedia representa la relación del hombre con el significante, pero lo deja atrapado en un destino fatal. Es decir, sujeto a la dimensión imaginaria del falo. 
En cambio, la comedia, por más que en el seno de la familia lleve las huellas de la tragedia, se convierte en la posibilidad, para el hombre y la mujer, de tomar una relación distinta con la palabra respecto de la que toma en la dimensión trágica.

Sostenemos que hay relación entre la comedia y el falo significante porque (...) "La comedia asume, recoge, goza de la relación con un efecto fundamentalmente relacionado con el orden significante, a saber, la aparición de aquel significado llamado el falo" (Lacan, 1957/1958, p. 270).

En una situación semejante a la comedia, gozar del significado del falo, es decir complacerse de asumir el lugar de hombre o de mujer, es efecto de la castración. Esta operación eleva el falo al estado significante, representante del deseo, como algo que se puede dar o retirar y no se confunde con la imagen, lo que permite gozar de la condición de sujeto en el hombre o mujer que representa. De esas representaciones resulta que la comedia hace aparecer en la escena las distintas funciones del ideal del yo vinculada con un símbolo erótico.

El símbolo erótico lo es del significante falo, el que se distingue del ideal.

El significante falo posee un carácter "universal” (Ravinovich, D. 2009, p.50), es el "marcador significante" (Assoun, P, 2005, p. 119) porque, reiteramos, da, en él, realidad al sujeto, irrealiza las relaciones que han de significarse entre los sexos, pero, por su lógica atributiva fálica, designa quién lo tiene y quién lo es. En cambio, los ideales están sujetos a lo que cada ser hablante significa como hombre o mujer, el significado del falo, en la demanda de amor cuyo límite es la cópula.

Con respecto a los ideales, Lacan en su Escrito "La significación del falo" formula que: "Estos ideales reciben su vigor de la demanda que tienen el poder de satisfacer, y que es siempre demanda de amor, con su complemento de la reducción del deseo a la demanda" (p. 661).

De la demanda de amor, el ideal toma la energía para parecer, ya sea protegiendo el tener o enmascarando la falta en el Otro, pero parecer tenerlo y serlo lleva al sujeto a la decepción. Al ir hacia el encuentro de la imagen ideal, el sujeto tropieza con lo cómico, lo ridículo en la relación de amor, porque se dirige al otro buscando el falo, pero esa búsqueda está sujeta al desengaño porque opera la castración, en tanto el ser está perdido y se trata de una sustitución.

Ahora bien, la decepción es la condición de posibilidad de la relación entre los sexos, porque el falo significante señala que el deseo, en tanto falta, es un límite al ideal del yo, y no queda capturado, ni reducido a la demanda. Es ese límite del que la risa deriva tras el acto de la copulación.

Por lo que se refiere al deseo en la copulación, Lacan (1962/1963) subraya la función del falo, menos phi, $(-\varphi)$ ligada a la caída del objeto, porque, en esta época de su enseñanza, redefine la noción de castración, cuya función se presenta en la caducidad de los objetos que tienen la cualidad de amboceptor. Luego, aborda, también, en la detumescencia, el orgasmo que se realiza normalmente, es decir como satisfacción común, porque revela una de las dimensiones de esta operación. Y nos dice:

(...)La detumescencia en la copulación merece nuestra atención porque pone de relieve una de las dimensiones de la castración. El hecho de que el falo sea más significativo en la vivencia humana por su posibi- 
lidad de ser un objeto caído que por su presencia- he aquí lo que designa la posibilidad del lugar de la castración en la historia del deseo. Es esencial ponerlo de relieve, porque de no distinguir estructuralmente el deseo de la dimensión del goce, y si no se plantea la cuestión de saber si hay para cada pareja una relación -y-cual entre el deseo, en particular el deseo del Otro, y el goce, todo el asunto está condenado a la oscuridad (Lacan, 1962/1963, pp. 182183).

A nuestro entender, la detumescencia es testimonio de una de las dimensiones de la castración que funciona con la privación. Porque, es importante subrayar que aquello que distingue el deseo del goce, ya que lo separa, es la función del objeto $a$ que se constituye como efecto del reconocimiento de la falta en el Otro, aunque, la relación al deseo del Otro es diferente en el hombre y la mujer. Entonces, si opera la castración, el objeto $a$, en tanto real que resiste a la asimilación del significante, representa el objeto perdido, dicho de otro modo, aquello que se pierde en la significantización, y se articula como causa de deseo. Por eso, el orgasmo, en la detumescencia, testimonia que la castración funciona a nivel del objeto $a$ causa de deseo.

Más adelante, en El Seminario. Libro 10. La Angustia, Lacan (1962/1963) escribe: (...) "Si el falo se presenta en la función de a con el signo menos, es porque funciona en la copulación humana, no sólo como instrumento del deseo, sino también como su negativo" (p.191).

La función del falo, no solo como instrumento sino como negativo del deseo, revela la insistencia de lo real, inaprensible por la significación, que señala la dimensión ilusoria del deseo en la copulación. Lo ilusorio en la cópula radica en que el deseo, en su tendencia a la satisfacción, se dirige a ese resto, $a$, que queda de la división del sujeto por el significante y que ningún falo puede sustituir. Por la función del menos phi $(-\varphi)$, la satisfacción en la copulación es siempre un falso final y una equivocación que sostiene la función del sujeto deseante en la relación erótica entre los sexos.

A ese falso final lo encontramos articulado a la pulsión de muerte, que irrumpe entre el deseo y la demanda, dado que la satisfacción señala un real que es como una pequeña muerte. Sin embargo, como la satisfacción es un falso final, ese real intrincado a la vida por la existencia del amor puede generar un sentimiento cómico al final de la copulación.

Por eso, cuando Lacan (1962/1963) se pregunta “¿qué es?” (p.284) lo que demandamos, la respuesta es la satisfacción de una demanda que se relaciona con la muerte, es decir, concierne a lo que, freudianamente hablando, es la tendencia al nirvana. Pero esa tendencia encuentra un tope en la demanda misma, porque la pulsión, también, tiene relación con la demanda de hacer el amor. Por lo que, el maestro francés nos dice: (...) "Lo que demandamos es morir, incluso morirnos de risa -no sin razón indico siempre que el amor participa de lo que llamo un sentimiento cómico" (p. 284).

Al final del orgasmo, lo cómico puede situarse en la risa. La risa es un efecto cómico que señala el límite que el deseo y la castración le imponen a la demanda.

En la enseñanza de Lacan (1962/1963) lo cómico surge por la existencia del amor que intrica a la vida, por la función del 
menos phi, ese real que queda como resto de satisfacción en el orgasmo. En la investigación de Freud (1905), el placer cómico resulta de "un gasto de representación (investidura) ahorrado” (p. 223).

Freud (1905) se aproxima al estudio de lo cómico en su obra El chiste y su relación con lo inconsciente. De esa extensa y rica investigación, subrayamos que, a diferencia del chiste, “que se hace” (p. 173), lo cómico "se descubre" (p. 173) en el psiquismo, y se produce como "un hallazgo no buscado" (p. 180) en el vínculo social.

En tanto "sustituto de lo perdido" (Freud, 1905, p. 212), discernimos lo cómico en la risa. Es un sentimiento que resulta de la diferencia de gasto de investidura ahorrado, entre el contraste de representaciones, y lo cómico vuelve, “a esa diferencia de gasto que se juzga superfluo" (p.206), susceptible de descarga y fuente de placer cuando no encuentra otro empleo.

Freud (1905) en el "El chiste y las variedades de lo cómico" afirma:

(...) Condición de la génesis de lo cómico es que nos veamos movidos a aplicar a la misma operación del representar, simultáneamente o en rápida sucesión, dos diversas maneras de representación entre las que sobreviene la «comparación» y surge como resultado la diferencia cómica. Tales diferencias de gasto surgen entre lo ajeno y lo propio, lo habitual y lo alterado, lo esperado y lo que sobreviene (p.221).

Por eso lo cómico, cercano al humor por su localización en el preconsciente, es un “recurso" (Freud, 1905, p. 216) con el que cuenta el aparato anímico para trami- tar esa diferencia de investidura sobrante que sobreviene de la comparación, y recuperar el placer que solo se pierde "por el propio desarrollo de la actividad" (p. 223). En efecto, podemos inferir en la risa al final del orgasmo la recuperación de ese placer que solo se pierde por el propio desarrollo de la copulación.

Para finalizar, formulamos que la risa, variedad de placer cómico, surge en el reposo posterior del orgasmo, y al velar la presentificación de lo real pulsional recubre la angustia de castración. La risa, entonces, revela que el falo, en tanto menos phi, $(-\varphi)$, funciona en sus equivalencias dado que es "un médium universal en el campo de la conjunción sexual" (Lacan, 1962/1963, p. 292) que sostiene la relación del hombre y la mujer porque su punto de encuentro es la exclusión de hallarlo entre los sexos.

\section{REFERENCIAS BIBLIOGRAFICAS}

Assoun, P. (2006) Lecciones psicoanaliticas sobre masculino y femenino, Buenos Aires, Argentina: Ediciones Nueva Visión.

Freud, S. (1979) "El chiste y las variedades de lo cómico", Obras Completas, Buenos Aires, Argentina: Amorrortu Editores, volumen (8), pp. 173-225. 
LaCan, J. (1994) EL Seminario. Libro 4. La Relación de Objeto, Buenos Aires, Argentina: Ediciones Paidós.

(1999) EL Seminario. Libro 5.

Las Formaciones del Inconsciente, Buenos Aires, Argentina: Ediciones Paidós. (2002) "La significación del falo", Escritos 2, Buenos Aires, Argentina: Siglo Veintiuno Editores, pp. 653662.

(2014) El Seminario. Libro 6. El deseo y su interpretación, Buenos Aires, Argentina: Ediciones Paidós.

(2003) EL Seminario. Libro 8. La Transferencia, Buenos Aires, Argentina: Ediciones Paidós.

(2006) EL Seminario. Libro10. La Angustia, Buenos Aires, Argentina: Ediciones Paidós.

(1987) EL Seminario. Libro11.

Los Cuatro Conceptos Fundamentales del Psicoanálisis, Buenos Aires, Argentina: Ediciones Paidós.

Rabinovich, D. (2009) Lectura De "La Significación Del Falo”, Buenos Aires, Argentina: Ediciones Manantial SRL.

\section{COMO CITAR ESTE ARTÍCUlO:}

Morelli, V (2019). El Falo entre los Sexos en Revista Psicoanálisis en la Universidad $N^{\circ} 3$. Rosario, Argentina. UNR editora.

Verónica Morelli

Psicóloga y Profesora de Psicología. Magíster en Psicoanálisis. Jefe de Trabajos Prácticos en la cátedra Psicoanálisis y Psicopatología de la Facultad de Psicología. UNR. Docente e investigadora con categoría cinco (5). Atención clínica en consultorio privado. 